\title{
Does Diastolic Dysfunction Cause Exertional Dyspnea in Newly Diagnosed Hyperthyroid Patients with Preserved Left Ventricular Systolic Function?
}

\author{
Khadije Mohammadi ${ }^{1}$, Zahra Davoudi ${ }^{1}$, Azam Erfanifar ${ }^{1}$, Fatemeh Karamali ${ }^{1}$, Sajad \\ Erami $^{2}$, Hooman Bakhshandeh Abkenar ${ }^{3}$, and Maryam Shojaeifard ${ }^{3}$ \\ ${ }^{1}$ Affiliation not available \\ ${ }^{2}$ Shahid Sadoughi University of Medical Sciences and Health Services \\ ${ }^{3}$ Rajaie Cardiovascular Medical and Research Center
}

April 28, 2020

\begin{abstract}
Abstract Background: diastolic dysfunction has been reported as a cardiovascular effect of hyperthyroidism, and is also supposed to be a cause of heart failure manifestations in the patients with preserved ejection fraction (EF). Methods: for evaluation of diastolic function in hyperthyroid patients and also diastolic Function during performing exercise stress echocardiography, we conducted this study on 26 newly diagnosed hyperthyroid patients compared to 26 healthy subjects as control group. Diastolic function of the patients at rest was assessed using these parameters as follows: left atrium volume index, tricuspid regurgitation (TR) velocity, mitral inflow early diastolic flow (E wave), tissue doppler of mitral annular velocity (e'), E/e' ratio, and isovolumic relaxation time (IVRT); and during exercise stress echocardiography, we judged diastolic response by measuring E/e' ratio and TR velocity at the peak of stress in terms of the American Society of Echocardiography guideline. Results: the mean age of the patients was $39.77 \pm 12.0$ years old and $53.8 \%$ of them were female. Cardiovascular symptoms among the patients were the followings: palpitation (46.2\%), exertional dyspnea (19.2\%), and atypical chest pain (7.7\%). All the patients had normal diastolic function at the time of resting and there was no significant difference in diastolic parameters between the two groups except for IVRT, which was lower in hyperthyroid patients. Interestingly, no patients developed diastolic dysfunction during exercise stress echocardiography. Conclusion: our finding did not support Hyperthyroidsm associated diastolic dysfunction as a cause of exertional intolerance and dyspnea in the patients with preserved EF.
\end{abstract}

\section{Introduction}

Hyperthyroidism is defined as a clinical condition resulted from abnormal high serum thyroid hormones levels (1). Any increase in the level of thyroid hormone leads to change in many systems of human body including cardiovascular system, because Thyroid hormone acts through genomic and non-genomic mechanisms to regulate cardiac function.(2) Cardiomyocytes have thyroid hormone nuclear receptors that when bound alter gene transcription, can regulate intracellular calcium metabolism and myofilaments. Nongenomic effects on cardiomyocytes include altering ion channel permeability and then leading to tachycardia. (3)

Hemodynamic effects of hyperthyroidism increased heart rate (HR); reduced peripheral vascular resistance; and increased preload, stroke volume, and consequently cardiac output. (4). Thus cardiovascular manifestation are common among the patients and hyperthyroid patients may clinically develop some symptoms like exertional dyspnea, palpitation, orthopnea, paroxysmal nocturnal dyspnea and peripheral edema.(1, 2)

More common cardiac manifestations of hyperthyroidism that was documented in various studies are systolic hypertension, left ventricular(LV) hypertrophy, increased LV mass, heart failure, and cardiac arrhythmia 
including atrial fibrillation $(1,2,5,6)$; however, evaluation of left ventricular diastolic function in hyperthyroidism showed conflicting results in different studies.(7-11) accordingly, in some of them, diastolic dysfunction was supposed as a cause of heart failure symptoms in the patients with preserved EF $(7,12)$, while in others, the enhanced diastolic function was observed.(10) Considering that frequently, symptom of diastolic dysfunction only occurs during exercise, as LV filling pressure is normal at rest, but it is increased with exercise.(13) So, exercise stress echocardiography is a useful modality to detect diastolic capacity reserve by providing insight to cardiovascular hemodynamic. In this study, we tried to assess diastolic function in the hyperthyroid patients using the latest guideline of American society of echocardiography (ASE) (14) and also evaluation of diastolic function in response to exercise during stress echocardiography to see whether it cause exertional dyspnea or not.

\section{Methods}

This case-control study was conducted on 26 newly diagnosed and untreated hyperthyroid patients in comparison to 26 healthy. Also, age and sex were matched in subjects as control group in Rajaie Heart center echo lab. Patients were referred to us from out-patient clinic of Loghman Hakim Hospital endocrinology department. The recruitment phase was done from January 2019 to January 2020, and the patients who were diagnosed with hyperthyroid based on clinical and laboratory data, had no history of hypertension, diabetes mellitus, cardiovascular disease, hyperlipidemia, anemia, pulmonary, and neuromuscular disease, and take no medication were entered to the study. Control subjects were chosen among the people with normal stress echocardiography result who came to our echo lab for checkup or non-anginal chest pain and had no past medical history with normal lab test for thyroid function, lipid profile, blood sugar, and hemoglobin.

At first, we assessed the patients by electrocardiography (ECG) and conventional echocardiography, and exclude those who had valvular heart disease, any structural heart disease, left ventricular (LV) systolic dysfunction by means of LV ejection fraction of less than $55 \%$ by Simpson method, pulmonary hypertension (systolic pulmonary artery pressure more than $35 \mathrm{mmHg}$ using tricuspid regurgitation (TR) velocity), and non-sinus rhythm. Three of 29 patients who were referred to our center, were excluded from the study due to LV dysfunction, pulmonary hypertension, and atrial fibrillation plus LV dysfunction.

\section{Stress echocardiography}

A complete two dimensional(2D) and Doppler echocardiography was done by an experienced operator at the time of resting in left lateral decubitus position, using the same machine (affinity 70 Philips with 1$5 \mathrm{MHz}$ transducer) in terms of the American society of echocardiography recommendation. LV end diastolic volume, end systolic volume, and EF were calculated from apical two and four chamber views based on the modified Simpson method. Diastolic parameters including mitral inflow velocities (E and A waves), mitral annulus tissue Doppler velocities (septal and lateral e'), left atrium volume index (LAVI), and peak TR velocity were measured by averaging in three consecutive cardiac cycle to estimate diastolic function in terms of the 2016 ASE guideline(14). Isovolumic relaxation time (IVRT) and myocardial performance index (MPI) were also measured using tissue Doppler method. Then, the patient and control groups underwent an exercise stress echocardiography on treadmill using Bruce protocol. End points for exercise were chest pain, dyspnea, exhaustion, target heart rate of more than $90 \%$ adjusted by age, and significant ST segment deviation. Blood pressure and ECG were recorded at any stage. Peak stress images including 5 standard echocardiographic views (parasternal long and short axis views, apical 4 chamber, 3 chamber, and 2 chamber views) were obtained immediately after cessation of test during one minute for evaluation of ischemia and speckle tracking strain analysis. TR peak velocity was obtained within 1 minute from peak stress, and $\mathrm{E}$ and e' velocities were measured at time of 60-90 milliseconds from peak when E and A, e' and a' waves were not fused in heart rate about 120 beat per minute.

\section{Definition}

-Hyperthyroidism was diagnosed when serum free Thyroxin (T4) and triiodothyronine (T3) were more than upper limit of laboratory range, and Thyroid stimulating hormone (TSH) was less than $0.1 \mathrm{mIU} / \mathrm{mL}$. (T3 and T4 were measured by radioimmunoassay and TSH by immunometric method using commercially available 
kits)

-Based on the ASE guideline, for evaluation of diastolic function in the patients with preserved EF we considered four criteria including: 1) septal e' velocity $<7 \mathrm{~cm} / \mathrm{s}$ or lateral e' $<10 \mathrm{~cm} / \mathrm{s}, 2$ ) average E/e' ratio $>14$, 3) $\mathrm{LAVI}>34 \mathrm{ml} / \mathrm{m} 2$, and 4) peak TR velocity $>2.8 \mathrm{~m} / \mathrm{s}$. Diastolic function was normal when more than half of four variables were negative. LV diastolic dysfunction was present when more than $50 \%$ of indices were positive, and diastolic function was indeterminate if half of parameters were positive.

-Stress echocardiography test was considered to be positive for diastolic dysfunction when all of following parameters were present in peak stress: 1 ) average E/e' $>14$ or septal E/e' $>15,2$ ) peak TR velocity $>2.8 \mathrm{~m} / \mathrm{s}$, and 3) septal e' velocity $<7 \mathrm{~cm} / \mathrm{s}$ or lateral velocity $<10 \mathrm{~cm} / \mathrm{s}$ at base line.

-IVRT and MPI were calculated by tissue doppler method when myocardial velocities were recorded using spectral pulse doppler from mitral annular level in apical 4 chamber view. MPI was calculated as sum of isovolumic relaxation time and isovolumic contraction time were divided by ejection time.

Statistical analysis: The results of quantitative variables with normal distribution were expressed as mean and standard deviation (mean $\pm \mathrm{SD}$ ), and numerical variables without normal distribution were expressed as median with inter-quartile range (IQR). Qualitative variables were reported by number and percentage. To compare the numerical variables with and without normal distribution, "independent samples t-test" and "Mann-Whitney U test" were used, respectively. Also, Chi-Square test was used to compare nominal

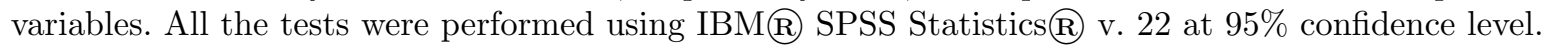

\section{Results}

26 newly diagnosed hyperthyroid patients and 26 healthy subjects as control group were enrolled in this study. Mean age of the hyperthyroid patients was $39.77 \pm 12.0$ years old and $53.8 \%$ (n: 14) of them were females. The etiology of hyperthyroidism was Graves $(80.76 \%)$, toxic multi nodular goiter $(11.54 \%)$, toxic adenoma $(3.85 \%)$, and sub-acute thyroiditis $(3.85 \%)$. cardiovascular symptoms and signs include the followings: palpitation (46.2\%), exertional dyspnea (19.2\%), and atypical chest pain (7.7\%). Baseline characteristics of the hyperthyroid patients are listed in table-1.

Table-1: Baseline characteristics of the hyperthyroid patients,

Characteristics

Age, mean \pm SD (years old) Female sex, n (\%) BSA(m2) TSH, mean (range) Duration of symptoms(month), median (IQR)

SD: Standard deviation, n: Number, BSA: body surface area

In comparison between hyperthyroid and control groups, there was no significant difference in clinical parameters such as age, sex, and body surface area (BSA), and two groups were matched. Hemodynamic parameters assessment showed that, the hyperthyroid patients had a higher heart rate compared to control group at rest, but both systolic and diastolic blood pressures were not significantly different between the two groups. All the subjects were in sinus rhythm without any significant ST segment deviation.

In echocardiographic evaluation of diastolic function, we found that, no patient had septal e' $<7$ or lateral $\mathrm{e}^{\prime}<10 \mathrm{~cm} / \mathrm{s}$ and mean values were $9.74 \pm 2.1$ and $13.43 \pm 2.9$, respectively. Mean E/e' at rest in the hyperthyroid patients was $7.2 \pm 1.1$ that was in normal range and also was not elevated in none of the individuals. Also, TR velocity and LAVI met criteria for diastolic dysfunction in no patient; so, regarding the ASE guideline definition, no one had diastolic dysfunction at rest. (Table-2)

Diastolic indices including septal and lateral mitral annular velocities, TR velocity, E/e' ratio, LAVI, MPI, and PAP showed no notable difference between the two groups; IVRT was the only parameter that was lower in the hyperthyroid patients compared to the control group. (P value: 0.008 ; Table- 2 ) 
Table -2. Comparison of clinical characteristic, hemodynamic, and echocardiographic parameters at rest between the hyperthyroid and control groups

E/e' Maximal exercise $\Delta$ E/e' TR velocity $(\mathrm{m} / \mathrm{s})$ Maximal exercise $\Delta$ TR velocity PAP (mmHg) Maximal exercise $\Delta$ PAP I

Bpm: beat per minute, SBP: Systolic blood pressure, DBP: Diastolic blood pressure

During performing stress echocardiography, all subjects completed stress test in view of reaching at least $90 \%$ of maximum predicted heart rate, and no one had developed evidence of ischemia in echocardiography or ECG. About 7 patients (26.9\%) reported mild dyspnea at peak stress that was not restricting for exercise and no one had typical chest pain and dizziness. No significant ST-T change and arrhythmia were induced.

For evaluation of diastolic function reserve, according to the mentioned criteria in previous section, no one had developed criteria of diastolic dysfunction in stress echocardiography and mean E/e' ratio and TR velocity were $7.25 \pm 1.4$ and 2.6(2.39-2.88), respectively. There was also no significant difference between the two groups. (Table-3)

Table -3. Comparison of echocardiographic diastolic parameters at maximal exercise between the hyperthyroid and control groups.

E/e' Maximal exercise $\Delta$ E/e' TR velocity $(\mathrm{m} / \mathrm{s})$ Maximal exercise $\Delta$ TR velocity PAP (mmHg) Maximal exercise $\Delta$ PAP I

\section{Discussion}

Cardiovascular symptom such as exertional dyspnea and impaired exercise tolerance are common in the hyperthyroid patients. $(1,15)$. In our study, $19.2 \%$ of the patients had a history of exertional dyspnea. Probable explanations for exertional symptoms include ineffective oxygen utilization, respiratory muscle weakness and increased ventilator drive to breath, increased airway resistance, diminished lung compliance, and heart failure. (15) In Raphael's study (2), heart failure with the reduced EF was seen in $6 \%$ and heart failure with the preserved EF was seen in $10 \%$ of the patients. Yue et al. also reported heart failure presentation in $5.8 \%$ of the hyperthyroid patients that only in $50 \%$ of them, there was reduced EF (16), and they proposed that, diastolic dysfunction is the reason of HF symptoms in others.

We found that, no hyperthyroid patient had diastolic dysfunction that was in contrast with many previous studies, because Diastolic dysfunction specially impaired relaxation (grade 1) was frequently reported in many previous studies evaluated the hyperthyroid patients $(1,2,4,7,17)$. The most important explanation for this discrepancy was that, we used newer guideline criteria for defining diastolic dysfunction compared to previous studies that were based on the conventional indices, and also we excluded the patients with comorbidities that may affect diastolic function such as those with diabetes, hypertension, and Coronary artery disease. We included newly diagnosed hyperthyroid patients who received no medication and this point was another difference between our study and previous studies that were done on the patients consuming anti-thyroid medications, and suggested that, maybe initiation of drugs induced diastolic change that need to be assessed in future studies.

By comparing diastolic parameters between the two groups, IVRT was the only index that significantly differed and other indices including E/e', LAVI, TR velocity, septal, and lateral e' were comparable. Enhanced diastolic function was also reported in Mintz G's study (10), and their results about IVRT was similar to us, however their study was done only at rest. Shorter IVRT in hyperthyroidism is due to lusitropic effect of thyroxin (18) through positively regulated sarcoplasmic Ca-ATPase because Reuptake of calcium into the 
sarcoplasmic reticulum early in diastole can determine the rate of the left ventricle relaxation (isovolumic relaxation time).

In our study, diastolic function reserve was also assessed by exercise stress echocardiography using ASE guideline criteria. Accordingly, this evaluation was not performed in previous studies, and interestingly, we found that no one in hyperthyroid group had developed diastolic dysfunction criteria.

Conclusion : our finding did not support Hyperthyroidism associated diastolic dysfunction as a cause of exertional intolerance and dyspnea in the patients with preserved EF and normal PAP.

\section{Limitations}

First, by considering that we excluded the patients with any comorbidity and those taken medications, the main limitation of our study was the small sample size. Second, we selected our cases from outpatient clinic and did not include more severe cases who may need hospitalization and Probably with more severe cardiac involvement including diastolic dysfunction.

Author contribution : Khadije Mohammadi (concept, data collection, analysis and interpretation, drafting article), Zahra Davoudi MD (data collection), Azam Erfanifar MD(data collection), Fatemeh Karamali MD (data collection), Sajad Erami MD (data collection), Hooman Bakhshandeh MD, PHD (statistics), Maryam Shojaeifard MD (design, data interpretation and critical revision of article)

\section{References}

1. Mulatu HA. Pattern and Presentation of Thyro-Cardiac Disease among Patients with Hyperthyroidism Attending a Tertiary Hospital in Ethiopia: A Cross Sectional Study. Ethiopian journal of health sciences. 2019;29(1).

2. Anakwue RC, Onwubere BJ, Ikeh V, Anisiuba B, Ike S, Anakwue A-MC. Echocardiographic assessment of left ventricular function in thyrotoxicosis and implications for the therapeutics of thyrotoxic cardiac disease. Therapeutics and clinical risk management. 2015;11:189.

3. Otto CM. The practice of clinical echocardiography: Elsevier Health Sciences; 2007.

4. Teasdale SL, Inder WJ, Stowasser M, Stanton T. Hyperdynamic right heart function in Graves' hyperthyroidism measured by echocardiography normalises on restoration of euthyroidism. Heart, Lung and Circulation. 2017;26(6):580-5.

5. Osuna PM, Udovcic M, Sharma MD. Hyperthyroidism and the Heart. Methodist DeBakey cardiovascular journal. 2017;13(2):60.

6. Klein I, Danzi S. Thyroid disease and the heart. Circulation. 2007;116(15):1725-35.

7. Yue WS, Chong BH, Zhang XH, Liao SY, Jim MH, Kung AW, et al. Hyperthyroidism-induced left ventricular diastolic dysfunction: implication in hyperthyroidism-related heart failure. Clinical Endocrinology. 2011;74(5):636-43.

8. Jing X, Liu Y, Huang H. Left ventricular diastolic function of patients with newly diagnosed hyperthyroidism. Sichuan da xue xue bao Yi xue ban= Journal of Sichuan University Medical science edition. 2012;43(3):462-6.

9. Di Bello V, Aghini-Lombardi F, Monzani F, Talini E, Antonangeli L, Palagi C, et al. Early abnormalities of left ventricular myocardial characteristics associated with subclinical hyperthyroidism. Journal of endocrinological investigation. 2007;30(7):564-71.

10. Mintz G, Pizzarello R, KLEIN I. Enhanced left ventricular diastolic function in hyperthyroidism: noninvasive assessment and response to treatment. The Journal of Clinical Endocrinology \& Metabolism. 1991;73(1):146-50. 
11. Smit J, Eustatia-Rutten C, Corssmit E, Pereira A, Frolich M, Bleeker G, et al. Reversible diastolic dysfunction after long-term exogenous subclinical hyperthyroidism: a randomized, placebo-controlled study. The Journal of Clinical Endocrinology \& Metabolism. 2005;90(11):6041-7.

12. Biondi B. Heart failure and thyroid dysfunction. Eur J Endocrinol. 2012;167(5):609-18.

13. Ha J-W, Andersen OS, Smiseth OA. Diastolic stress test: invasive and noninvasive testing. JACC: Cardiovascular Imaging. 2019.

14. Nagueh SF, Smiseth OA, Appleton CP, Byrd BF, Dokainish H, Edvardsen T, et al. Recommendations for the evaluation of left ventricular diastolic function by echocardiography: an update from the American Society of Echocardiography and the European Association of Cardiovascular Imaging. European Journal of Echocardiography. 2016;17(12):1321-60.

15. Kahaly GJ, Wagner S, Nieswandt J, Mohr-Kahaly S, Ryan TJ. Stress echocardiography in hyperthyroidism. The Journal of Clinical Endocrinology \& Metabolism. 1999;84(7):2308-13.

16. Siu C-W, Yeung C-Y, Lau C-P, Kung AW, Tse H-F. Incidence, clinical characteristics and outcome of congestive heart failure as the initial presentation in patients with primary hyperthyroidism. Heart. 2007;93(4):483-7.

17. Abdulrahman RM, Delgado V, Ng AC, Ewe SH, Bertini M, Holman ER, et al. Abnormal cardiac contractility in long-term exogenous subclinical hyperthyroid patients as demonstrated by two-dimensional echocardiography speckle tracking imaging. European journal of endocrinology. 2010;163(3):435.

18. Lilly LS, Braunwald E. Braunwald's heart disease: a textbook of cardiovascular medicine: Elsevier Health Sciences; 2012. 